\title{
Distribution distance-based threshold auto-tuning method for switching median filter
}

\author{
Ryosuke Kubota $^{1 \mathrm{a})}$ and Noriaki Suetake ${ }^{2 \mathrm{~b})}$ \\ ${ }^{1}$ Department of Intelligent System Engineering, Ube National College of Technology \\ 2-14-1 Tokiwadai, Ube 755-8555, Japan \\ ${ }^{2}$ Graduate School of Science and Engineering, Yamaguchi University \\ 1677-1 Yoshida, Yamaguchi 753-8512, Japan \\ a) kubota@ube-k.ac.jp \\ b) suetake@sci.yamaguchi-u.ac.jp
}

\begin{abstract}
In this paper, we propose a new tuning method of threshold, which is a parameter of a switching median filter, by using the distribution distance. The switching median filter detects noise-corrupted pixels based on a threshold, and restores only the detected pixels. The present switching median filter deals with the random-valued impulse noises, whose distribution is ideally assumed as a uniform. In the present method, the threshold is tuned by evaluating a distribution distance between the assumed and the detected noises. $L_{1}$-norm is used here as the distribution distance. The effectiveness of the proposed method is verified by some experiments.
\end{abstract}

Keywords: switching median filter, random-valued impulse noise, parameter tuning, distribution distance, $L_{1}$-norm

Classification: Science and engineering for electronics

\section{References}

[1] T. Chen, K.-K. Ma, and L.-H. Chen, "Tri-state median filter for image denoising," IEEE Trans. Image Process., vol. 8, no. 12, pp. 1834-1838, Dec. 1999.

[2] A. Bovik, Handbook of Image and Video Processing, Academic Press, San Diego, 2000.

[3] T. Chen and H. R. Wu, "Space variant median filters for the restoration of impulse noise corrupted images," IEEE Trans. Circuits Syst. II, Analog Digit. Signal Process., vol. 48, no. 8, pp. 784-789, Aug. 2001.

[4] T. Sun and Y. Neuvo, "Detail-preserving median based filters in image processing," Pattern Recognit. Lett., vol. 15, no. 4, pp. 341-347, April 1994.

[5] R. Pandey, "An improved switching median filter for uniformly distributed impulse noise removal," Proc. World Academy of Science, Engineering and Tech., Johannesburg, South Africa, pp. 349-351, Feb. 2008.

[6] X. Zhang, Z. Yin, and Y. Xiong, "Adaptive switching mean filter for impulse noise removal," 2008 IEEE Congress on Image and Signal Pro- 
cessing, Sanya, China, pp. 275-278, May 2008.

[7] G. H. Golub and C. F. Van Loan, Book Matrix Computations, The Johns Hopkins University Press, Baltimore, 1996.

\section{Introduction}

Quality of digital images is needed to be high in many research and application fields. However, digital image is often corrupted by the impulse noise in the image sensing and/or transmission processes. In order to realize a fine restoration of the image corrupted by the impulse noise, various nonlinear filters based on the median filter have been studied so far $[1,2,3]$.

Especially, a switching median filter [4] has been frequently employed and studied in recent years $[5,6]$. The typical switching median filters include a noise detector based on a threshold, and carries out the median filtering to the only detected noisy pixels. In order to effectively use the switching median filter, its detecting threshold has to be appropriately tuned for the signal of concern. However, the tuning of the threshold has been achieved empirically up to the present. Therefore, it is needed to develop the tuning method of the threshold, which can determine the value effectively and efficiently without any trial and error.

To determine the effective threshold automatically, we propose a new tuning method based on the distribution distance between a noise model assumed beforehand and the noise signals detected by the detector. As the distribution distance, $L_{1}$-norm is employed. Concretely, the tuning is realized by finding the threshold giving the minimum $L_{1}$-norm. The effectiveness and the validity of the proposed method are illustrated by some experiments.

\section{Proposed threshold tuning method of switching median fil- ter}

In this paper, we consider the most popular model of the random-valued impulse noise. This model takes into account a bit error in the digitalized signal transmission. A signal $x(i, j)$ corrupted with the random-valued impulse noise is then represented by:

$$
x(i, j)= \begin{cases}s(i, j), & \text { probability } 1-p, \\ h, & \text { probability } p,\end{cases}
$$

where, $s(i, j)$ is the original signal, and takes 256 level ( 8 bit) values. $p$ represents a noise occurrence probability. The noise-corrupted pixel value $h$ takes from 0 to 255 depending on a uniform distribution, because each bit in $s(i, j)$ inverts randomly.

A detailed-preserving median based filter [4] is a typical switching median filter. In this method, the pixel value $x(i, j)$ is detected as the impulse noise, when $x(i, j)$ satisfies:

$$
|x(i, j)-m(i, j)| \geq \varepsilon,
$$


where $m(i, j)$ stands for the output signal at the pixel $(i, j)$ by the ordinary median filter. $\varepsilon$ is a threshold. The switching median filter carries out the median filtering for the only detected pixels.

In the proposed method, the threshold $\varepsilon$ is tuned based on a distribution distance between a noise model assumed beforehand and the noise detected by the detector. When the distribution distance is small, it is judged as a fine noise detection is achieved. In the proposed method, the optimal value $\varepsilon^{*}$ is concretely obtained as follows:

$$
\varepsilon^{*}=\arg \min _{\varepsilon} D\left(H_{d,(\varepsilon)}, H_{a}\right),
$$

where $D$ is a function to calculate the distribution distance. $H_{d,(\varepsilon)}$ stands for a probability density function of the noises detected with the threshold $\varepsilon$. The probability density function is then obtained from a normalized histogram of a set of $x(i, j)$ detected as the noise. $H_{a}$ indicates the probability density function of the assumed random-valued impulse noise. In the proposed method, the assumption on the distribution of the noise is imposed by the model of Eq. (1). Therefore, $H_{a}$ can be assumed as a uniform distribution in a range $[0,255]$.

As the $D$ in Eq. (3), some metrics, e.g., Hellinger distance, JensenShannon divergence, symmetric Kullback-Leibler divergence and so on, can be considered to use. In this paper, we focus on $L_{1}$-norm [7], which is defined by:

$$
L_{1}\left(H_{d,(\varepsilon)}, H_{a}\right)=\sum_{\ell=0}^{255}\left|H_{d,(\varepsilon)}(\ell)-H_{a}(\ell)\right|
$$

because its calculation is very simple. $H_{a}(\ell)$, which is assumed as the uniform distribution, is $\frac{1}{256}$ for arbitrary $\ell$. $L_{1}$-norm becomes small when the two distributions are similar each other.

\section{Experimental results}

The effectiveness and the validity of the proposed method are verified by experiments employing some test images, which were taken by digital cameras. An example of the test images is shown in Fig. 1(a), which constitutes of $1,459 \times 1,094$ pixels. In the experiments, the test images are corrupted by the random-valued impulse noise with $p=0.03,0.05,0.1,0.15$ and 0.2. Fig. 1 (b) shows the normalized histogram of the random-valued impulse noises superimposed on Fig. 1(a). As shown in Fig. 1(b), it is observed that the noise distribution is almost uniform.

A mean square error (MSE) is used as an index for the quantitative evaluation. MSE is calculated from the original noise-free image of Fig. 1 (a) and the output image restored by the switching median filter. MSE thus becomes small when the restored image is similar to the original one. Fig. 1 (c) shows the MSE obtained by changing $\varepsilon$ of the switching median filter. For each curve, the $\varepsilon$ giving the minimum MSE in Fig. 1 (c) denotes here the optimal value $\varepsilon_{\text {opt }}$. From Fig. 1 (c), it can be said that $\varepsilon_{\mathrm{opt}} \mathrm{s}$ take various values depending on the noise occurrence probability. 


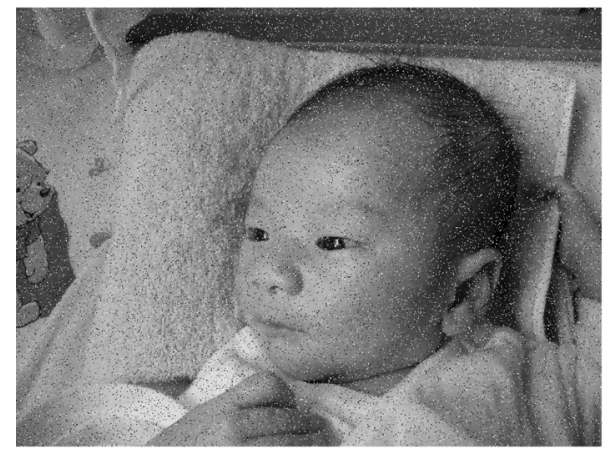

(a)

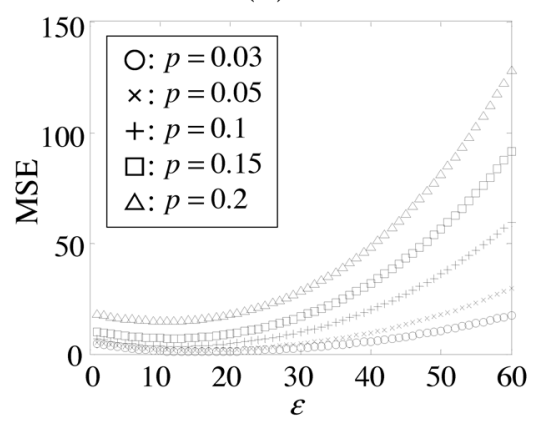

(c)

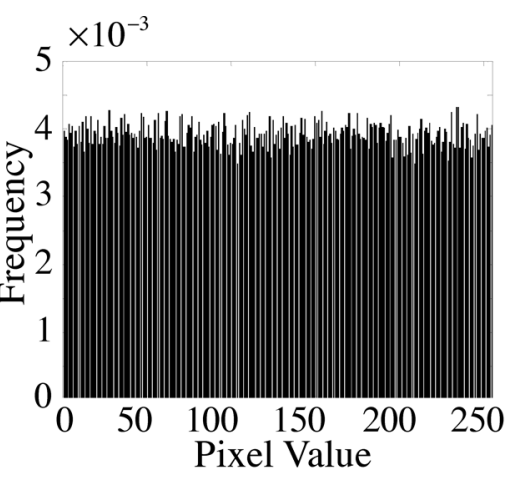

(b)

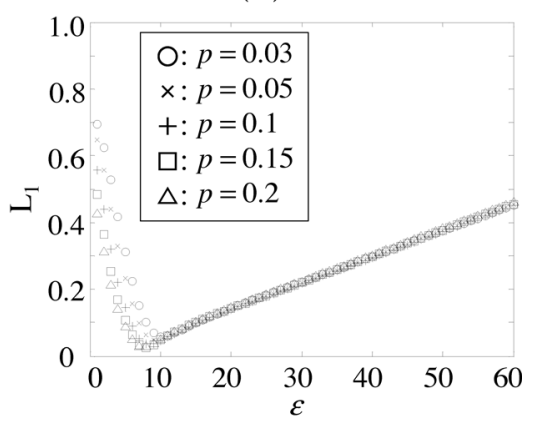

(d)

Fig. 1. Examples of test image and relationships: (a) test image $(p=0.1)$, (b) normalized histogram of the noises in (a), (c) $\varepsilon$ vs. MSE, (d) $\varepsilon$ vs. $L_{1}$-norm.

On the other hand, Fig. 1 (d) shows the $L_{1}$-norm between the assumed noise model and the normalized histogram of the noises detected by the detector with various $\varepsilon$. The $\varepsilon$ giving the minimum $L_{1}$-norm in Fig. 1 (d) stands for the tuned value $\varepsilon^{*}$. As shown in Figs. 1 (c) and (d), $\varepsilon^{*}$ s obtained based on distribution distance are sufficiently similar to $\varepsilon_{\text {opt }}$ although $\varepsilon^{*}$ s are little bit different from $\varepsilon_{\mathrm{opt}} \mathrm{s}$. We have also confirmed that the relationships $\varepsilon$ vs. other distribution distances (Hellinger distance, Jensen-Shannon divergence, symmetric Kullback-Leibler divergence) are equivalent to the relationship $\varepsilon$ vs. $L_{1}$. From these results, it seems that the distribution distance-based threshold tuning method highly promises a secure performance.

Experimental results of four test images including Fig. 1(a) with some noise probabilities are shown in Table I. In this table, $D^{*}$ represents the minimum $L_{1}$-norm. $\varepsilon^{*}$ and $\varepsilon_{\text {opt }}$ are the obtained threshold based on $L_{1}$-norm and the optimal threshold based on MSE, respectively. MSE* and $\mathrm{MSE}_{\mathrm{opt}}$ stand for the mean square errors between the original noise-free image and the output image restored by the switching median filter with $\varepsilon^{*}$ and $\varepsilon_{\mathrm{opt}}$, respectively.

From Table I, the proposed threshold tuning is good for the most test images although $\varepsilon^{*}$ is not perfectly same to $\varepsilon_{\mathrm{opt}}$. Especially, from Table I (c), it can be said that the proposed method performs well. In the cases where Image 2 is used as input image of concern, however, differences between $\mathrm{MSE}_{\mathrm{opt}} \mathrm{S}$ and the MSE*s are especially large comparing with the cases where 
Table I. Experimental result of each image.

(a) Image 1 (Fig. 1 (a))

\begin{tabular}{c|c|c|c|c|c}
\hline$p$ & $D^{*}$ & $\varepsilon^{*}$ & $\varepsilon_{\mathrm{opt}}$ & $\mathrm{MSE}^{*}$ & $\mathrm{MSE}_{\mathrm{opt}}$ \\
\hline 0.03 & 0.059 & 11 & 15 & 1.5 & 1.0 \\
\hline 0.05 & 0.054 & 10 & 14 & 2.1 & 1.6 \\
\hline 0.1 & 0.039 & 9 & 13 & 3.9 & 3.4 \\
\hline 0.15 & 0.023 & 8 & 12 & 7.6 & 7.0 \\
\hline 0.2 & 0.029 & 8 & 11 & 15.1 & 14.6 \\
\hline
\end{tabular}

(b) Image 2

\begin{tabular}{c|c|c|c|c|c}
\hline$p$ & $D^{*}$ & $\varepsilon^{*}$ & $\varepsilon_{\mathrm{opt}}$ & $\mathrm{MSE}^{*}$ & $\mathrm{MSE}_{\mathrm{opt}}$ \\
\hline 0.03 & 0.052 & 20 & 29 & 8.1 & 7.0 \\
\hline 0.05 & 0.040 & 17 & 25 & 10.7 & 9.6 \\
\hline 0.1 & 0.031 & 15 & 20 & 17.2 & 16.4 \\
\hline 0.15 & 0.030 & 14 & 18 & 26.9 & 26.4 \\
\hline 0.2 & 0.031 & 13 & 16 & 44.1 & 43.9 \\
\hline
\end{tabular}

(c) Image 3

\begin{tabular}{c|c|c|c|c|c}
\hline$p$ & $D^{*}$ & $\varepsilon^{*}$ & $\varepsilon_{\mathrm{opt}}$ & $\mathrm{MSE}^{*}$ & $\mathrm{MSE}_{\mathrm{opt}}$ \\
\hline 0.03 & 0.058 & 6 & 8 & 0.2 & 0.2 \\
\hline 0.05 & 0.042 & 6 & 7 & 0.4 & 0.3 \\
\hline 0.1 & 0.035 & 5 & 7 & 1.8 & 1.7 \\
\hline 0.15 & 0.028 & 5 & 6 & 7.8 & 7.7 \\
\hline 0.2 & 0.021 & 5 & 6 & 25.5 & 25.4 \\
\hline
\end{tabular}

(d) Image 4

\begin{tabular}{c|c|c|c|c|c}
\hline$p$ & $D^{*}$ & $\varepsilon^{*}$ & $\varepsilon_{\mathrm{opt}}$ & $\mathrm{MSE}^{*}$ & $\mathrm{MSE}_{\mathrm{opt}}$ \\
\hline 0.03 & 0.058 & 10 & 15 & 1.4 & 1.2 \\
\hline 0.05 & 0.049 & 10 & 13 & 1.9 & 1.7 \\
\hline 0.1 & 0.036 & 8 & 11 & 3.9 & 3.9 \\
\hline 0.15 & 0.026 & 8 & 11 & 7.2 & 6.9 \\
\hline 0.2 & 0.027 & 8 & 10 & 14.7 & 14.4 \\
\hline
\end{tabular}

other images are used. It is supposed that there are many detail parts in Image 2 and the detection of the noise is difficult inherently.

Figs. 2 (c) and (d) show the parts of Image 1 (Fig. 1 (a)) restored by the switching median filtering with the thresholds obtained based on the MSE and the proposed tuning, respectively. It is observed that the restoration image by the proposed method is the almost same to that by the MSE-based optimal tuning. Moreover, Figs. 2 (e) and (f) show the normalized histograms obtained from the MSE-based and the proposed methods, respectively. From Figs. 2 (e) and (f), it can be said that the distribution of the noises detected by the proposed tuning is more similar to that of the input noises shown in Fig. 1(b), when the proposed tuning is compared to by the MSE-based optimal tuning. From these results, the effectiveness and validity of the proposed method are confirmed. 


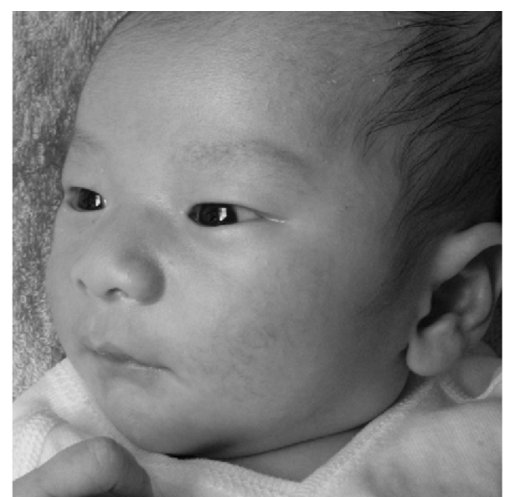

(a)

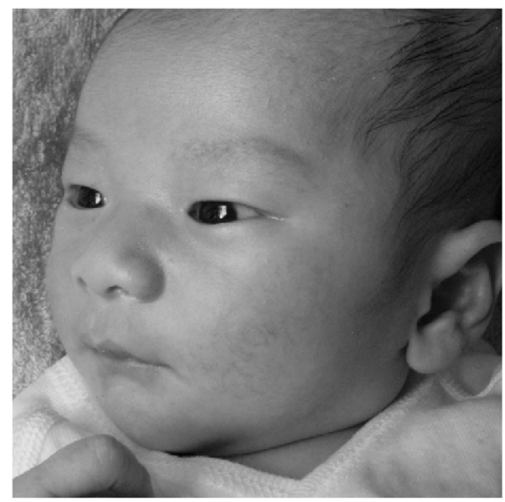

(c)

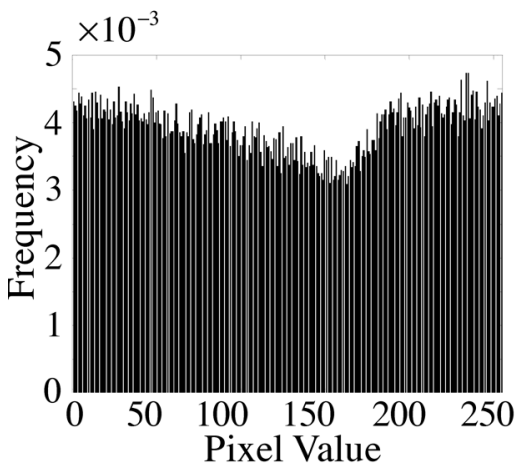

(e)

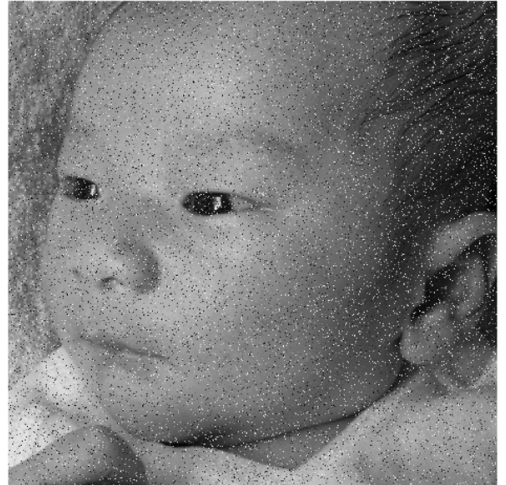

(b)

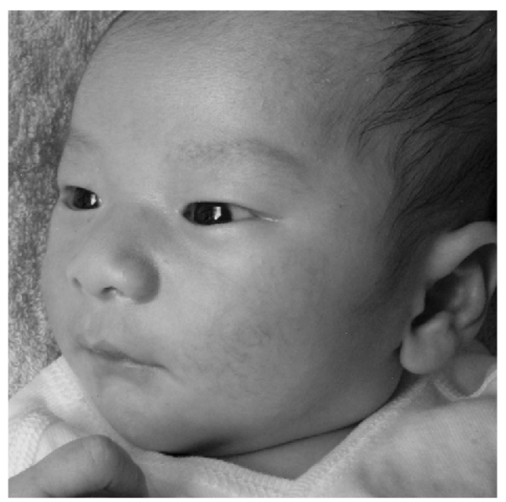

(d)

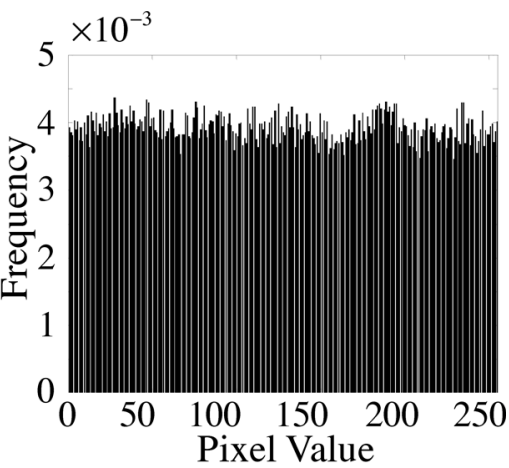

(f)

Fig. 2. Experimental results for Image 1 (partial image of Fig. 1(a)): (a) original image, (b) noisy input image $(p=0.1),(\mathrm{c})$ output image by the MSE-based optimal tuning, (d) output image by the proposed tuning, (e) normalized histogram of noises detected by the MSE-based tuning, (f) normalized histogram of noises detected by the proposed tuning.

\section{Conclusion}

In this paper, we proposed a new threshold tuning method for the switching median filter using a distance between signal distributions. The appropriate thresholds for the various images were obtained by the present method without any trial and error. Through the experimental results, the effectiveness 
and the validity of the present method were illustrated.

Future works are the development of an efficient search algorithm to find the optimal threshold, and its application to the color images. 